\title{
Urban nestlings have reduced number of feathers in Great Tits Parus major
}

\author{
KRISZTINA SÁNDOR, ${ }^{1,2 *}$ ANDRÁS LIKER, ${ }^{1,3}$ CSENGE SINKOVICS,${ }^{1}$ ÁRON PÉTER ${ }^{4}$ \& \\ GÁBOR SERESS ${ }^{3}$ \\ ${ }^{I}$ Behavioural Ecology Research Group, Center for Natural Sciences, University of Pannonia, \\ Veszprém, Hungary \\ ${ }^{2}$ MTA-ELTE Comparative Ethology Research Group, Budapest, Hungary \\ ${ }^{3}$ MTA-PE Evolutionary Ecology Research Group, University of Pannonia, Veszprém, Hungary \\ ${ }^{4}$ Department of Parasitology and Parasitic Diseases, Faculty of Veterinary Medicine, University of \\ Agricultural Sciences and Veterinary Medicine, Cluj-Napoca, Romania \\ * Corresponding author \\ Email: s.krisztinaa@gmail.com
}

The plumage of birds plays an essential role in thermal insulation and influences the heat tolerance of birds.

These plumage functions are mainly determined by the number and the density of feathers, but it is unclear how feather density responds to environmental changes in wild populations. In urban birds, both high temperature and limited food could generate changes in plumage traits. To investigate the effect of urbanization, we compared the number of feathers in nestlings between urban and forest Great Tits Parus major using a novel non-invasive method. We showed that urban nestlings have fewer feathers than forest nestlings at 6-9 days old. Although the density of feathers was slightly higher in urban nestlings, this was the result of the smaller size of their feather tracts. We suggest that the reduced feather number may be the result of either adaptation to higher urban temperatures, constrained feather development due to limited optimal nestling-food sources in urban environments, or both. Concentrating body feathers in a reduced tract area may also help birds to adapt to higher urban temperatures because this can increase the relative size of bare body surfaces which may facilitate heat dissipation. We suggest several possibilities for future studies that would help to disentangle the underlying mechanisms responsible for the observed patterns.

Keywords: plumage, heat tolerance, heat dissipation, food availability, urban heat island 
In birds, besides a range of physiological, behavioural, and some morphological mechanisms (Tattersall et al. 2012, Ryeland et al. 2017, Thompson et al. 2018), the plumage also plays a key role in regulating body temperature by facilitating or constraining the extent to which heat is retained close to the body (Wolf \& Walsberg 2000). This, in turn, is determined by several properties of the plumage, such as the structure, number, and density of body feathers (Wolf \& Walsberg 2000, Jiang et al. 2010, Grémillet et al. 2012). Feathers grow in distinct feather tracts (Clench 1970) in which the formation of feather follicles takes place during embryonic development (Yu et al. 2004), so the maximum number of feathers that a bird can develop is fixed at hatching. Interspecific comparative studies have demonstrated that variation in the number and density of feathers is related to habitat type, diet (Osváth et al. 2018), body size (Hutt 1938, Møller 2015), and migration distance (Møller 2015). Two recent studies also suggest negative correlations between ambient temperature of the species' environment, feather number (Møller 2015) and feather density (Osváth et al. 2018). However, much less is known about how these traits vary within a species. Experimental work in captive poultry suggests that both food availability for egg-laying females (Dahlke et al. 2008) and incubation temperature of eggs (Scott et al. 2015) affect the number of follicles formed in embryos. While the few within-species studies on wild birds also suggest the influence of temperature and food availability on feather structure (Pap et al. 2008, Broggi et al. 2011), it is virtually unexplored how other plumage traits like feather number and density differ between wild populations living under different environmental conditions.

Urbanization is a major form of global anthropogenic landscape conversion. The associated changes (e.g., altered microclimate and food availability, increased pollution, and human disturbance) could be strong drivers of fast evolutionary responses in cities, making urban habitats an ideal setting to study the adaptability of species to rapid ecological changes (Rivkin et al. 2019, Liker 2020). Urban areas, for example, have strongly altered microclimate (Cui \& Shi 2012) and food availability (Robb et al. 2008, Seress et al. 2018), which, in turn, may influence the thermoregulatory properties of body feathers. In the temperate zone, the ambient temperature is often several degrees higher in cities compared to their surroundings (urban heat island effect, UHI; Oke 1982), resulting in more frequent heat days and increased risk of heat stress (Ward et al. 2016), but also in milder winters (Hinkel et al. 2003). This altered temperature regime in cities 
may affect urban birds' survival either detrimentally (due to the increased risk of heat stress, especially in early life stages) or favourably (due to the increased overwinter survival in adults), depending for example on life stage. Indeed, the effects of the UHI have already been documented in a wide range of organisms (Parris \& Hazell 2005, Jochner \& Menzel 2015, Diamond et al. 2017, Merckx et al. 2018) including birds (Pipoly et al. 2013, Rodríguez \& Barba 2016). Recent studies also found adaptive responses to the elevated temperature in some urban populations (Brans et al. 2017, Diamond et al. 2017), but such responses in avian plumage have not yet been described or quantified.

When compared to more natural areas, urban environments often harbour a reduced abundance of arthropods (Jones \& Leather 2012, New 2015, Fenoglio et al. 2020), which may also be of lower quality (e.g., lower carotenoid content in caterpillars at urban versus rural sites; Isaksson \& Andersson 2007) and have smaller body size (Merckx et al. 2018), resulting in a relative scarcity of high-quality food sources (e.g., lepidopteran larvae; Narango et al. 2018, Seress et al. 2018, 2020, Jarrett et al. 2020) for urban insectivorous birds. On the other hand, cities offer large amounts of low-quality, easily accessible anthropogenic food which is readily consumed by birds (Robb et al. 2008). This altered food palette, however, may influence the quality and number of developing body feathers, for example through its effects on the pre-breeding condition of egg-laying females (for similar carry-over effects of food quality on egg and nestling quality see Plummer et al. 2013a, 2013b). While suboptimal food is known to affect, for example, plumage coloration in wild birds (Eeva et al. 2009, Isaksson 2009), its impacts on the thermoregulatory properties of feathers are unexplored.

Here, we compared the number and density of contour feathers between nestlings at two urban and two forest habitats that differ in their ambient temperature and natural food supply (see Methods: Fieldwork). To achieve this, we developed a novel method that is, contrary to the former studies, non-invasive (i.e., it does not require the plucking of birds' feathers) and can be applied on living specimens (nestlings at an early age). We predicted fewer feathers and lower feather density in urban compared to non-urban nestlings for at least two, mutually non-exclusive reasons. First, both traits might be adaptive to UHI (e.g., to decrease the risk of heat stress during the nestling stage (Speakman \& Król 2010)), and second, feather development of offspring may be constrained by the suboptimal feeding conditions experienced by egg-laying females in urban environments (see above). As a model organism, we studied the Great Tit Parus major. This is a 
common species in urban and natural habitats across the Western Palearctic (Gosler \& Clement 2007), and is non-migratory in our study region and thus affected by local factors (food availability, temperature) throughout its life cycle.

\section{METHODS}

Fieldwork

We carried out our study in April-May 2018 in two urban and two forest nestbox-breeding populations of Great Tits in Hungary (Fig. S1. The urban study sites were located in the cities of Balatonfüred

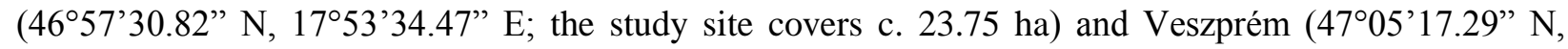
$17^{\circ} 54^{\prime} 29.66$ " E; the study site covers c. 43.59 ha), where the nestboxes (a total of 66 and 77 nestboxes) were placed in university campuses, public parks, and a cemetery. The vegetation of these sites consists of both native and introduced plant species. The forest study sites were located in deciduous woodlands near Szentgál village $\left(47^{\circ} 06^{\prime} 039.75^{\prime \prime} \mathrm{N}, 17^{\circ} 41^{\prime} 017.94^{\prime}\right.$ E; the study site covers c. 51.30 ha; a total of 92 nestboxes) characterized mainly by European Beech Fagus sylvatica and European Hornbeam Carpinus betulus, and in Vilma-puszta $\left(47^{\circ} 05^{\prime} 002.74^{\prime \prime} \mathrm{N}, 17^{\circ} 52^{\prime} 001.28^{\prime}\right.$ ' E, the study plot covers c. 48.10 ha; a total of 110 nesboxes), characterized mainly by Downy Oak Quercus cerris and South European Flowering Ash

\section{Fraxinus ornus.}

From egg-laying, we monitored nestboxes at least twice per week (and more frequently during the expected time of hatching) to determine the exact hatching date (day of hatching = day 1 ). We collected feather number data when nestlings were 6-9-days old (urban nestlings (mean \pm SE): $7.4 \pm 0.1 \mathrm{~d}$, forest nestlings: $7.3 \pm 0.1 \mathrm{~d}$ ). This age range represents an optimal developmental stage for this measurement because feathers have already emerged from the skin within the main feather tracts (pers. obs.) but they are still covered by sheaths, so feathers do not overlap and can be counted accurately (Fig. 1a). To further increase the precision of our measurements, on the day of sampling we always chose nestlings that were in the optimal feather development stage for the purpose of our measurements. Then, in each brood, we selected three nestlings and took close-up photographs on their sternal region of the ventral feather tract by gently stretching chicks by their legs and heads (Fig. 1a). Before taking photographs, we attached a millimeter scale $(20 \times 10 \mathrm{~mm})$ to the nestlings' belly next to the examined feather tract (Fig. 1a); this label contained 
nestling ID and served as a reference scale during photo processing (see below). When taking photographs, the camera lens was always parallel with the scale and the feather tract. Nestling body mass was measured with a Pesola spring balance $( \pm 0.1 \mathrm{~g})$. We sampled only three nestlings per brood because this procedure took several minutes per nestling, which would have meant too much disturbance for large broods.

\section{Measuring feather traits on photographs}

To extract data from the photographs we used ImageJ (v. 1.51). First, we determined the scale for each photo by using the attached millimeter scale. Second, we outlined the area of the ventral feather tract as follows (Fig. 1a). At the neck, we determined its boundary by following the feather tips of the crosswise feather row, starting at the point where the feather tract of the wing intersects with the ventral feather tract (Fig. 1b). On the sternal side, the boundary followed the feather tips of the $7^{\text {th }}$ row of feathers (rows counted from the lateral edge of the feather tract, Fig. 1c). Finally, at the lateral and the posterior ends of the tract we followed the tips of the most exterior feathers (Fig. 1a). Next, within this area, we counted the number of contour feathers (henceforth 'feather number'), and also measured the size of the outlined body surface area ( $\mathrm{mm}^{2}$, henceforth 'area') and the length of the three longest feathers ( $\mathrm{mm}$, henceforth 'feather length'). Then we calculated the density of contour feathers as feather number divided by the area (henceforth 'feather density'). The final sample involved 95 urban (Veszprém $n=60$, Balatonfüred $n=35$ ) and 73 forest (Szentgál $n=54$, Vilma-puszta $n=19$ ) nestlings (from 42 urban and 29 forest broods; for details, see SOM: Feather measurements).

\section{Statistical analyses}

First, we tested between- and within-measurer repeatability by intraclass correlation (ICC, package 'irr'). The repeatability was high for all variables both between- and within observer (SOM: Feather measurements). Next, to test our predictions for the effects of habitat type on feather tract traits, we followed the approach suggested by Ruxton and Beauchamp (2008) and conducted pre-planned comparisons between the study sites in two steps (see also e.g. Pipoly et al. 2019; Seress et al. 2020). In the first step, we identified significant confounding variables besides the effect of the main predictor (study site). Thus, we fitted separate linear mixed-effect models (LME, package ' $n l m e$ ', with Gaussian error distribution) for 
each of three nestling feather traits (feather number, feather density and ventral tract area), with individual nestlings as replicates, while also controlling for potential confounding variables (detailed in Table 1.).

Table 1. The structure and description of the three mixed-effect models (LME) fitted for nestlings' feather traits.

\begin{tabular}{|c|c|c|}
\hline $\begin{array}{l}\text { Response } \\
\text { variable }\end{array}$ & Explanatory variables & Comments \\
\hline \multicolumn{3}{|c|}{ Feather number } \\
\hline & Study site (four levels) & main effect \\
\hline & Body mass $(\mathrm{g})$ & to control for the condition of nestlings \\
\hline & Feather length (mm) & $\begin{array}{l}\text { to control for its effect on the accuracy of measurements } \\
\text { (as longer feathers may partially overlap) }\end{array}$ \\
\hline & Clutch size (max. nr. of eggs) & $\begin{array}{l}\text { to control for the potential trade-off between brood size } \\
\text { and embryo quality }\end{array}$ \\
\hline & Nest ID & random factor \\
\hline \multicolumn{3}{|c|}{ Feather density (the $n r$. of contour feathers $/ \mathrm{mm}^{2}$ ) } \\
\hline & Study site (four levels) & main effect \\
\hline & Body mass $(g)$ & to control for the body size of nestlings \\
\hline & Feather length (mm) & $\begin{array}{l}\text { to control for its effect on area measurements (as the } \\
\text { feather tract was outlined along the feather tips) }\end{array}$ \\
\hline & Brood size (max. nr. of nestlings) & $\begin{array}{l}\text { to control for the potential trade-off between brood size } \\
\text { and nestling quality }\end{array}$ \\
\hline & Nest ID & random factor \\
\hline \multicolumn{3}{|l|}{ Area $\left(\mathbf{m m}^{2}\right)$} \\
\hline & Study site (four levels) & main effect \\
\hline & Body mass $(\mathrm{g})$ & to control for the body size of nestlings \\
\hline & Feather length $(\mathrm{mm})$ & $\begin{array}{l}\text { to control for its effect on area measurements (as the } \\
\text { feather tract was outlined along the feather tips) }\end{array}$ \\
\hline & Brood size (max. nr. of nestlings) & $\begin{array}{l}\text { to control for the potential trade-off between brood size } \\
\text { and nestling quality }\end{array}$ \\
\hline & Nest ID & random factor \\
\hline
\end{tabular}

150

In the second step, we conducted pre-planned comparisons to compare the means of the three response variables between habitats. We used separate linear models for each response variable that contained study site (four levels), and any significant $(P<0.05)$ predictors from the full LME models described above. Note that all the included covariates were statistically significant (Table 2a), except for clutch size in the feather number model $\left(A N O V A, \chi_{(I)}^{2}=0.267, P=0.605\right)$ and brood size in the area $\left(\chi_{(I)}^{2}\right.$ $=0.643, P=0.423)$ and feather density $\left(\chi_{(I)}^{2}=0.394, P=0.530\right)$ models, and these were thus not retained 
in the final models. Then we calculated linear contrasts from each model's estimated marginal means (package 'emmeans') between the two habitat groups (i.e., differences between the two urban and the two forest sites).

We conducted all analyses in R (v. 3.6.2; R Core Team 2019). We checked assumptions of normality and homogeneity of variance of residuals by inspecting the residual plots for each model, and also calculated VIF values (package 'car'), which indicated low collinearity between variables (VIF<2.18) in all cases.

\section{RESULTS}

The LME models indicated that all feather traits differed significantly between study sites (Table 2a, Fig. 2.). Linear contrast analyses also confirmed habitat differences: urban versus forest nestlings had significantly lower number of feathers (contrast $\pm \mathrm{SE}:-2.91 \pm 0.87, t=-3.35, P=0.001$ ) and higher feather density (contrast $\pm \mathrm{SE}: 0.08 \pm 0.04$ feather $/ \mathrm{mm}^{2}, t=2.08, P=0.041$ ) in the ventral feather tract. Body mass had a significant positive, and feather length had a negative effect on feather number, while feather density was negatively affected by both body mass and feather length (Table 2a). We also found smaller ventral feather tract areas in urban nestlings and this difference was significant after controlling for the effects of body mass and feather length (contrast $\pm \mathrm{SE}: 3.59 \pm 1.58, t=2.270, P=0.026$ ). Ventral feather tract area was significantly correlated with both body mass $\left(\chi_{(1)}^{2}=30.523, P<0.001\right)$ and feather length $\left(\chi_{(1)}^{2}=112.633, P<0.001\right)$.

\section{DISCUSSION}

In urban areas, ecological factors such as climate or food quality are significantly altered, and these changes can contribute to the differences in traits between urban and non-urban animal populations, whether through adaptation or developmental constraints. In this study, we compared feather numbers and density (i.e., traits that can strongly influence the insulation properties of plumage) between two urban and two forest habitats that greatly differ in ambient temperature and natural food supply. To our knowledge, this is the first study that has investigated the between-population variation of these traits in a wild bird species, and the first to 
have done so in an urbanization context. To achieve this we developed a novel, non-invasive, and easily applicable method with high repeatability that, contrary to former methods, can be applied to living birds. In comparison with earlier, more opportunistic sampling methods (e.g. Osváth et al. 2018), this new approach allows more systematic data collection from larger samples of birds.

Our result that nestlings had significantly fewer feathers in the urban (i.e., warmer) environment, is in line with our expectations and also corroborates the findings of Møller (2015) who showed a negative correlation between feather number and seasonal temperature (that is, birds have more feathers during autumn and winter, followed by a gradual feather loss during spring and summer until the next annual moult). Several mechanisms can contribute to reduced feather numbers in urban birds, as detailed below. Firstly, because feather density is a heritable trait (e.g., a moderate heritability was found in domestic chickens (Sun et al. 2019), fewer feathers could reflect genetic adaptation to higher ambient temperatures in urban relative to non-urban areas (as in our study system; SOM: Study sites). For example, it is well-known in poultry that fewer feathers are advantageous under high temperatures (Deeb \& Cahaner 1999), allowing easier heat dissipation, and enhancing heat stress tolerance (Jiang et al. 2010). On the same basis, increased ability to dissipate heat might also be beneficial for wild urban birds. This might especially hold for altricial bird species such as Great Tits, whose nestlings are exposed to the prevailing ambient temperatures in the nest, and do not have established competent thermoregulation in their early life so might be sensitive to the harmful effects of UHI during summer heat days (Rodríguez \& Barba 2016, Andreasson et al. 2018). As Pipoly et al. (2020) showed, heat days are indeed significantly more frequent at our urban compared to forest sites during the chick-rearing period: in 2013-2018 (six years) $45.9 \%$ of the urban broods experienced at least one heat day (range: 1-13 heat days per brood), while this rate was only $20.8 \%$ in the forest sites (range: 1-5 heat days per brood). High temperatures can be especially problematic for holenesting species breeding in artificial nestboxes, as the temperature of the cavities and nestboxes can be up to several degrees higher than the ambient temperature (Maziarz et al. 2017). The microclimate of the nest can therefore further enhance the effects of the UHI. Although altricial nestlings are already capable of active heat dissipation by panting by $8-10$ days old (hence regulating their body temperature to some extent, Andreasson et al. 2018), this can be costly as panting is associated also with intense water loss (Wolf \& Walsberg 1996). In natural habitats, this cost can be effectively compensated by consuming food with high 
water content such as caterpillars (Zandt 1996). In urban areas, however, the scarcity of such prey items (Seress et al. 2018) might significantly increase the cost of this behaviour, thereby increasing the relative role of plumage in the dissipation of extra heat for urban nestlings. Brood size may also influence the capacity of nestlings to dissipate heat and thermoregulate because smaller broods can cope with high temperatures more effectively, conversely larger broods are more effective at low temperatures (Mertens 1977, Andreasson et al. 2016). In our urban Great Tit populations, similarly to other studies, broods are consistently smaller than in the forest populations (Seress et al. 2018) a pattern that was also present in the current study (mean $\pm \mathrm{SD}$, number of hatchlings, urban: $8.0 \pm 1.9$; forest: $10.3 \pm 1.9$; number of 14-16 days old nestlings, urban: $6.5 \pm 2.9$; forest: $9.4 \pm 2.4$ ). Thus, smaller brood size in urban habitats may also contribute to the thermal tolerance of nestlings, potentially helping them to cope with the elevated nest microclimate in cities. Having fewer feathers, however, could also be advantageous for adult urban birds when coping with elevated summer temperatures (Grémillet et al. 2012, Nilsson \& Nord 2018, Nord \& Nilsson 2018, Tapper et al. 2020) in cities, while the reduced insulation capacity is probably less costly in winters due to the relatively milder climate in temperate zone cities (Hinkel et al. 2003).

Secondly, fewer feathers could be the result of environmental constraints on embryo development, when the number of follicles (hence feather number) is determined (Yu et al. 2004). For example, due to the low availability of optimal food sources in cities (see SOM: Study sites), females may be constrained from laying high-quality eggs (Toledo et al. 2016), resulting in suboptimal nutrition for embryo development (Krist 2011), and hence affecting feather follicle formation (Scott et al. 2015). Note that growing fewer feathers can be beneficial in poor nutritional environments because it reduces the energetic costs of feather development, and this energy can be invested into other essential traits such as immune functions (Moreno-Rueda 2010). Finally, nest microclimate during incubation may also affect nestling phenotype through developmental plasticity, as suggested by a study of Blue Tits Cyanistes caeruleus that found reduced tarsus length in nestlings under experimentally elevated incubation temperatures (Nord \& Nilsson 2011). Thus, it is conceivable that higher incubation temperatures in urban nests could also contribute to the lower feather number of urban nestlings.

We found higher feather density in urban nestlings, which seems tocontradict our prediction and also the results of former studies documenting lower feather densities in warmer environments (Jiang et al. 
2010, Osváth et al. 2018). The higher feather density in urban nestlings, however, was the result of their smaller feather tract area. Importantly, this habitat difference in feather tract area remained significant even after we controlled for the effect of body mass, meaning that nestlings with the same weight had disproportionally smaller feather tract areas in urban compared to forest broods. In theory, one explanation for the observed habitat difference may be that body density is higher in urban than forest nestlings, for example, due to lower fat reserves. However, the broods involved in our study do not show habitat differences in fat score at ringing (SOM: Nestling fat score). Consequently, urban nestlings seem to have a relatively smaller feather-covered body surface compared to forest nestlings that may also be beneficial in terms of more effective heat dissipation (Speakman \& Król 2010, Grémillet et al. 2012, Nilsson \& Nord 2018). Thus, it is possible that urban birds can achieve increased heat tolerance not only by reduced feather numbers but also as a result of relatively larger bare body surfaces.

Finally, it is also important to note that at 6-9-days old, nestlings are still growing (Tilgar \& Mänd 2006). While their body size is increasing day by day, their feather number is not (as the number of feather follicles are fixed hatch, see the introduction), which may result in a gradual decrease in feather density through nestling growth. However, how these traits ultimately affect insulation and thus thermoregulation in adults, is still an open question and requires more research.

Although our study was correlative, it points to a previously undescribed effect of urbanization on bird morphology. Interestingly, our two urban study sites are relatively small and green, temperate-zone cities, and yet these areas, when compared to their surrounding natural habitats, are already sufficiently urbanized to exhibit significant differences in local climate and natural food supply as well as in the plumage traits of Great Tits. It may therefore be expected that the changes we found in feather number and density are more pronounced in more heavily urbanized regions, resulting in more important fitness consequences to 'big city birds'. Thus, in the future, it would be interesting to carry out similar studies in more urbanized areas or to measure the ratio of bare and feathered body surfaces of urban and non-urban conspecifics. Studying the effects of urbanization on the structural properties of contour feathers could also be an interesting next step. For example, changes in the density or proportion of the plumulaceous part in contour feathers (which also contributes to the thermal insulation of birds) could strengthen or, conversely, compensate for the effects that fewer feathers have on the thermoregulation of urban birds (Pap et al. 2017). 
Since both adaptation and constraints could explain our results, further experimental studies, such as food supplementation of egg-laying females, or heat treatment of eggs during the incubation period could help to disentangle the underlying mechanisms. Finally, it is also important to take into account that behavioural changes (Pattinson et al. 2020), smaller brood size (Mertens 1977), and body size (Merckx et al. 2018) may also contribute to help birds to tolerate elevated temperatures in urban habitats.

We thank Femke Cnossen, Krissie van den Heuvel, Anudari Batsaikhan and Kinga Kelemen for helping with fieldwork. This work was financed by the National Research Development and Innovation Office (NKFIH) of Hungary through the grant K132490 to AL. It was also supported by the TKP2020-IKA-07 project financed under the 2020-4.1.1-TKP2020 Thematic Excellence Programme by the National Research, Development and Innovation Fund of Hungary. ÁP was supported by the Colegium Talentum 2019 programme of Hungary.

\section{REFERENCES}

Andreasson, F., Nord, A. \& Nilsson, J.-Å. 2018. Experimentally increased nest temperature affects body temperature, growth and apparent survival in Blue Tit nestlings. J. Avian Biol. 49: jav-01620.

Brans, K.I., Jansen, M., Vanoverbeke, J., Tüzün, N., Stoks, R. \& De Meester, L. 2017. The heat is on: genetic adaptation to urbanization mediated by thermal tolerance and body size. Glob. Chang. Biol. 23: 5218-5227.

Broggi, J., Gamero, A., Hohtola, E., Orell, M. \& Nilsson, J.-Å. 2011. Interpopulation Variation in Contour Feather Structure Is Environmentally Determined in Great Tits. PLoS One 6: e24942.

Clench, M.H. 1970. Variability in body pterylosis, with special reference to the genus Passer. Auk 87: $650-691$.

Cui, L. \& Shi, J. 2012. Urbanization and its environmental effects in Shanghai, China. Urban Clim. 2: 115.

Dahlke, F., Gonzales, E., Furlan, R.L., Gadelha, A.C., Rosa, P.S., Meurer, R.F.P. \& Maiorka, A. 2008. Desenvolvimento folicular de embriões de frangos de corte de diferentes genótipos expostos ao estresse térmico crônico. Ciência Rural 38: 2314-2320. 
Deeb, N. \& Cahaner, A. 1999. The effects of naked neck genotypes, ambient temperature, and feeding status and their interactions on body temperature and performance of broilers. Poult. Sci. 78: 13411346.

Diamond, S.E., Chick, L., Perez, A.B.E., Strickler, S.A. \& Martin, R.A. 2017. Rapid evolution of ant thermal tolerance across an urban-rural temperature cline. Biol. J. Linn. Soc. 121: 248-257.

Eeva, T., Sillanpää, S. \& Salminen, J.P. 2009. The effects of diet quality and quantity on plumage colour and growth of Great Tit Parus major nestlings: A food manipulation experiment along a pollution gradient. J. Avian Biol. 40: 491-499.

Fenoglio, M.S., Rossetti, M.R. \& Videla, M. 2020. Negative effects of urbanization on terrestrial arthropod communities: A meta-analysis. Glob. Ecol. Biogeogr. 29: 1412-1429.

Gosler, A.G. \& Clement, P. 2007. Family Paridae (Tits and Chickadees). In: Handbook of the Birds of the World vol. 12. (J. del Hoyo, A. Elliott, \& D. A. Christei, eds), pp. 662-750. Barcelona.

Grémillet, D., Meslin, L. \& Lescroël, A. 2012. Heat dissipation limit theory and the evolution of avian functional traits in a warming world. Funct. Ecol. 26: 1001-1006.

Hinkel, K.M., Nelson, F.E., Klene, A.E. \& Bell, J.H. 2003. The urban heat island in winter at Barrow, Alaska. Int. J. Climatol. 23: 1889-1905.

Hutt, F.B. 1938. Number of feathers and body size in Passerine birds. Auk 55: 651-657.

Isaksson, C. 2009. The Chemical Pathway of Carotenoids: From Plants to Birds. Ardea 97: 125-128.

Isaksson, C. \& Andersson, S. 2007. Carotenoid diet and nestling provisioning in urban and rural great tits Parus major. J. Avian Biol. 38: 564-572.

Jarrett, C., Powell, L.L., McDevitt, H., Helm, B. \& Welch, A.J. 2020. Bitter fruits of hard labour: diet metabarcoding and telemetry reveal that urban songbirds travel further for lower-quality food. Oecologia 193: 377-388.

Jiang, R.S., Xia, W.T., Chen, X.Y., Geng, Z.Y. \& Hu, Z.Y. 2010. Density of contour feathers and heat tolerance in chickens. J. Appl. Anim. Res. 38: 253-255.

Jochner, S. \& Menzel, A. 2015. Urban phenological studies - past, present, future. Environ. Pollut. 203: $250-261$.

Jones, E.L. \& Leather, S.R. 2012. Invertebrates in urban areas: A review. Eur. J. Entomol. 109: 463- 
Krist, M. 2011. Egg size and offspring quality: A meta-analysis in birds. Biol. Rev. 86: 692-716.

Liker, A. 2020. Adaptive changes in urban populations. Biol. Futur. 1-8.

327

Maziarz, M., Broughton, R.K. \& Wesolowski, T. 2017. Microclimate in tree cavities and nest-boxes: Implications for hole-nesting birds. For. Ecol. Manage. 389: 306-313.

Merckx, T., Souffreau, C., Kaiser, A., Baardsen, L.F., Backeljau, T., Bonte, D., Brans, K.I., Cours, M., Dahirel, M., Debortoli, N., De Wolf, K., Engelen, J.M.T., Fontaneto, D., Gianuca, A.T., Govaert, L., Hendrickx, F., Higuti, J., Lens, L., Martens, K., Matheve, H., Matthysen, E., Piano, E., Sablon, R., Schön, I., Van Doninck, K., De Meester, L. \& Van Dyck, H. 2018. Bodysize shifts in aquatic and terrestrial urban communities. Nature 558: 113-116.

Mertens, J.A.L. 1977. Thermal conditions for successful breeding in Great Tits (Parus major L.). Oecologia 28: 31-56.

Møller, A.P. 2015. The allometry of number of feathers in birds changes seasonally. Avian Res. 6: 1-5.

Moreno-Rueda, G. 2010. Experimental test of a trade-off between moult and immune response in House Sparrows Passer domesticus. J. Evol. Biol. 23: 2229-2237.

Narango, D.L., Tallamy, D.W. \& Marra, P.P. 2018. Nonnative plants reduce population growth of an insectivorous bird. Proc. Natl. Acad. Sci. 115: 11549-11554.

New, T.R. 2015. Insect conservation and urban environments. Springer Cham.

Nilsson, J.Å. \& Nord, A. 2018. Testing the heat dissipation limit theory in a breeding passerine. Proc. $R$. Soc. B Biol. Sci. 285: 20180652.

Nord, A. \& Nilsson, J.-Å. 2011. Incubation Temperature Affects Growth and Energy Metabolism in Blue Tit Nestlings. Am. Nat. 178: 639-651.

Nord, A. \& Nilsson, J. 2018. Heat dissipation rate constrains reproductive investment in a wild bird. Funct. Ecol. 33: 250-259.

Oke, T.R. 1982. The energetic basis of the urban heat island. Q. Journal, R. Meteorol. Soc. 108: 1-24.

Osváth, G., Daubner, T., Dyke, G., Fuisz, T.I., Nord, A., Pénzes, J., Vargancsik, D., Vágási, C.I., Vincze, O. \& Pap, P.L. 2018. How feathered are birds? Environment predicts both the mass and density of body feathers. Funct. Ecol. 32: 701-712. 
Pap, P.L., Vágási, C.I., Czirják, G.Á. \& Barta, Z. 2008. Diet quality affects postnuptial molting and feather quality of the house sparrow (Passer domesticus): interaction with humoral immune function? Can. J. Zool. 86: 834-842.

Pap, P.L., Vincze, O., Wekerle, B., Daubner, T., Vágási, C.I., Nudds, R.L., Dyke, G.J. \& Osváth, G. 2017. A phylogenetic comparative analysis reveals correlations between body feather structure and habitat. Funct. Ecol. 31: 1241-1251.

Parris, K.M. \& Hazell, D.L. 2005. Biotic effects of climate change in urban environments: The case of the Grey-headed Flying-fox (Pteropus poliocephalus) in Melbourne, Australia. Biol. Conserv. 124: 267-276.

Pattinson, N.B., Thompson, M.L., Griego, M., Russell, G., Mitchell, N.J., Martin, R.O., Wolf, B.O., Smit, B., Cunningham, S.J., McKechnie, A.E. \& Hockey, P.A.R. 2020. Heat dissipation behaviour of birds in seasonally hot arid-zones: are there global patterns? J. Avian Biol. 51: jav.02350.

Pipoly, I., Bókony, V., Seress, G., Szabó, K. \& Liker, A. 2013. Effects of extreme weather on reproductive success in a temperate-breeding songbird. PLoS One 8: e80033.

Pipoly, I., Preiszner, B., Sándor, K., Sinkovics, C., Seress, G., Vincze, E., Bokony, V. \& Liker, A. 2020. Effects of extreme hot weather on the reproductive output of Great Tits (Parus major , L .) in urban and natural habitats. Preprint at: http://dx.doi.org/10.1101/2020.01.29.924332. BioRxiv 1-18.

Pipoly, I., Szabó, K., Bókony, V., Preiszner, B., Seress, G., Vincze, E., Schroeder, J. \& Liker, A. 2019. Higher frequency of extra-pair offspring in urban than forest broods of Great Tits (Parus major). Front. Ecol. Evol. 7: 1-5.

Plummer, K.E., Bearhop, S., Leech, D.I., Chamberlain, D.E. \& Blount, J.D. 2013a. Fat provisioning in winter impairs egg production during the following spring: A landscape-scale study of Blue Tits. J. Anim. Ecol. 82: 673-682.

Plummer, K.E., Bearhop, S., Leech, D.I., Chamberlain, D.E. \& Blount, J.D. 2013b. Winter food provisioning reduces future breeding performance in a wild bird. Sci. Rep. $\mathbf{3}$.

R Core Team. 2019. R: a language and environment for statistical computing. $R$ Foundation for Statistical Computing. Vienna, Austria. 
Rivkin, L.R., Santangelo, J.S., Alberti, M., Aronson, M.F.J., de Keyzer, C.W., Diamond, S.E., Fortin, M.J., Frazee, L.J., Gorton, A.J., Hendry, A.P., Liu, Y., Losos, J.B., MacIvor, J.S., Martin, R.A., McDonnell, M.J., Miles, L.S., Munshi-South, J., Ness, R.W., Newman, A.E.M., Stothart, M.R., Theodorou, P., Thompson, K.A., Verrelli, B.C., Whitehead, A., Winchell, K.M. \& Johnson, M.T.J. 2019. A roadmap for urban evolutionary ecology. Evol. Appl. 12: 384-398.

Robb, G.N., McDonald, R.A., Chamberlain, D.E. \& Bearhop, S. 2008. Food for thought: Supplementary feeding as a driver of ecological change in avian populations. Front. Ecol. Environ. 6: $476-484$.

Rodríguez, S. \& Barba, E. 2016. Nestling growth is impaired by heat stress: An experimental study in a mediterranean Great Tit population. Zool. Stud. 55.

Ruxton, G.D. \& Beauchamp, G. 2008. Time for some a priori thinking about post hoc testing. Behav. Ecol. 19: 690-693.

Ryeland, J., Weston, M.A. \& Symonds, M.R.E. 2017. Bill size mediates behavioural thermoregulation in birds. Funct. Ecol. 31: 885-893.

Scott, J., Da Costa, M.J. \& Oviedo-Rondón, E.O. 2015. Incubation temperature profiles affect broiler feathering. J. Appl. Poult. Res. 24: 49-57.

Seress, G., Hammer, T., Bókony, V., Vincze, E., Preiszner, B., Pipoly, I., Sinkovics, C., Evans, K.L. \& Liker, A. 2018. Impact of urbanization on abundance and phenology of caterpillars and consequences for breeding in an insectivorous bird. Ecol. Appl. 28: 1143-1156.

Seress, G., Sándor, K., Evans, K.L. \& Liker, A. 2020. Food availability limits avian reproduction in the city: An experimental study on great tits Parus major. J. Anim. Ecol. 89: 1570-1580.

Speakman, J.R. \& Król, E. 2010. Maximal heat dissipation capacity and hyperthermia risk: neglected key factors in the ecology of endotherms. J. Anim. Ecol. 79.

Sun, H., Hu, Y., Dou, T., Qu, L., Ma, M., Lu, J., Wang, X., Shen, M. \& Wang, K. 2019. Genetic architecture related to contour feathers density in an F2 resource population via a genome-wide association study. 3 Biotech 9: 1-6.

Tapper, S., Nocera, J.J. \& Burness, G. 2020. Heat dissipation capacity influences reproductive performance in an aerial insectivore. J. Exp. Biol. 223: jeb222232. 
Tattersall, G.J., Sinclair, B.J., Withers, P.C., Fields, P.A., Seebacher, F., Cooper, C.E. \& Maloney, S.K. 2012. Coping with thermal challenges: physiological adaptations to environmental temperatures. In: Comprehensive Physiology, pp. 2151-2202. John Wiley \& Sons, Inc., Hoboken, NJ, USA.

Thompson, M.L., Cunningham, S.J. \& McKechnie, A.E. 2018. Interspecific variation in avian thermoregulatory patterns and heat dissipation behaviours in a subtropical desert. Physiol. Behav. 188: $311-323$.

Tilgar, V. \& Mänd, R. 2006. Sibling growth patterns in Great Tits: Does increased selection on lasthatched chicks favour an asynchronous hatching strategy? Evol. Ecol. 20: 217-234.

Toledo, A., Andersson, M.N., Wang, H.L., Salmón, P., Watson, H., Burdge, G.C. \& Isaksson, C. 2016. Fatty acid profiles of Great Tit (Parus major) eggs differ between urban and rural habitats, but not between coniferous and deciduous forests. Sci Nat 103: 55 .

Ward, K., Lauf, S., Kleinschmit, B. \& Endlicher, W. 2016. Heat waves and urban heat islands in Europe: A review of relevant drivers. Sci. Total Environ. 569-570: 527-539.

Wolf, B.O. \& Walsberg, G.E. 1996. Respiratory and cutaneous evaporative water loss at high environmental temperatures in a small bird. J. Exp. Biol. 199: 451-457.

Wolf, B.O. \& Walsberg, G.E. 2000. The role of the plumage in heat transfer processes of birds. Am. Zool. 40: 575-584.

Yu, M., Yue, Z., Wu, P., Wu, D.Y., Mayer, J.A., Medina, M., Widelitz, R.B., Jiang, T.X. \& Chuong, C.M. 2004. The developmental biology of feather follicles. Int. J. Dev. Biol. 48: 181-191.

Zandt, H.S. 1996. Water content of prey of nestling Blue Tits in a Corsican habitat. Netherlands J. Zool. 47: $125-131$.

\section{Data availability statement}

The data that support the findings of this study are available from the corresponding author upon reasonable request. 


\section{Supporting information}

437 Additional supporting information can be found online in the Supporting Information section.

$438 \quad$ Figure S1. A map showing the location of and the distances between the four study sites.

439 Figure S2. Description of the study sites: the figure shows the differences in long-term ambient 440 temperature, caterpillar biomass (food availability), and nestlings' feather number between the two forest 441 and two urban sites. 
Table 2. Results of (a) the LME models of factors significantly affecting feather number and density

$444\left(\right.$ number $\left./ \mathrm{mm}^{2}\right)$ in Great Tit nestlings, and (b) habitat differences in these traits. The linear contrasts compare 445 the two forest sites to the two urban sites, positive values indicate higher values in forest nestlings. 446 Statistically significant $(\mathrm{P}<0.05)$ differences are highlighted in bold.

447

\begin{tabular}{|c|c|c|c|c|c|c|c|}
\hline & \multicolumn{3}{|c|}{ (a) Final models } & \multicolumn{4}{|c|}{ (b) Forest - urban contrasts } \\
\hline & $D F$ & $\chi^{2}$ & P-value & contrast \pm se & $D F$ & tratio & P-value \\
\hline \multicolumn{8}{|l|}{ Feather number } \\
\hline Study site & 3 & 24.228 & $<0.001$ & $2.91 \pm 0.87$ & 67 & 3.347 & 0.001 \\
\hline Body mass & 1 & 6.151 & 0.013 & & & & \\
\hline Feather length & 1 & 7.104 & 0.007 & & & & \\
\hline \multicolumn{8}{|l|}{ Density } \\
\hline Study site & 3 & 10.580 & 0.014 & $-0.08 \pm 0.039$ & 67 & -2.082 & 0.041 \\
\hline Body mass & 1 & 24.427 & $<0.001$ & & & & \\
\hline Feather length & 1 & 113.617 & $<0.001$ & & & & \\
\hline
\end{tabular}

Fig. 1. Measuring feather traits on photographs. (a) The examined area of a nestling's ventral feather tract 451 with the millimetre scale as reference. (b) The position of the anterior boundary (white line) is determined 452 by the intersection of the wing's feather tract with the ventral feather tract (white arrow) and follows the 453 feather tips of the crosswise feather row (white ellipse). (c) The sternal boundary (white line) was 454 determined at the tips of the $7^{\text {th }}$ row of feathers counted from the lateral side of the feather tract.
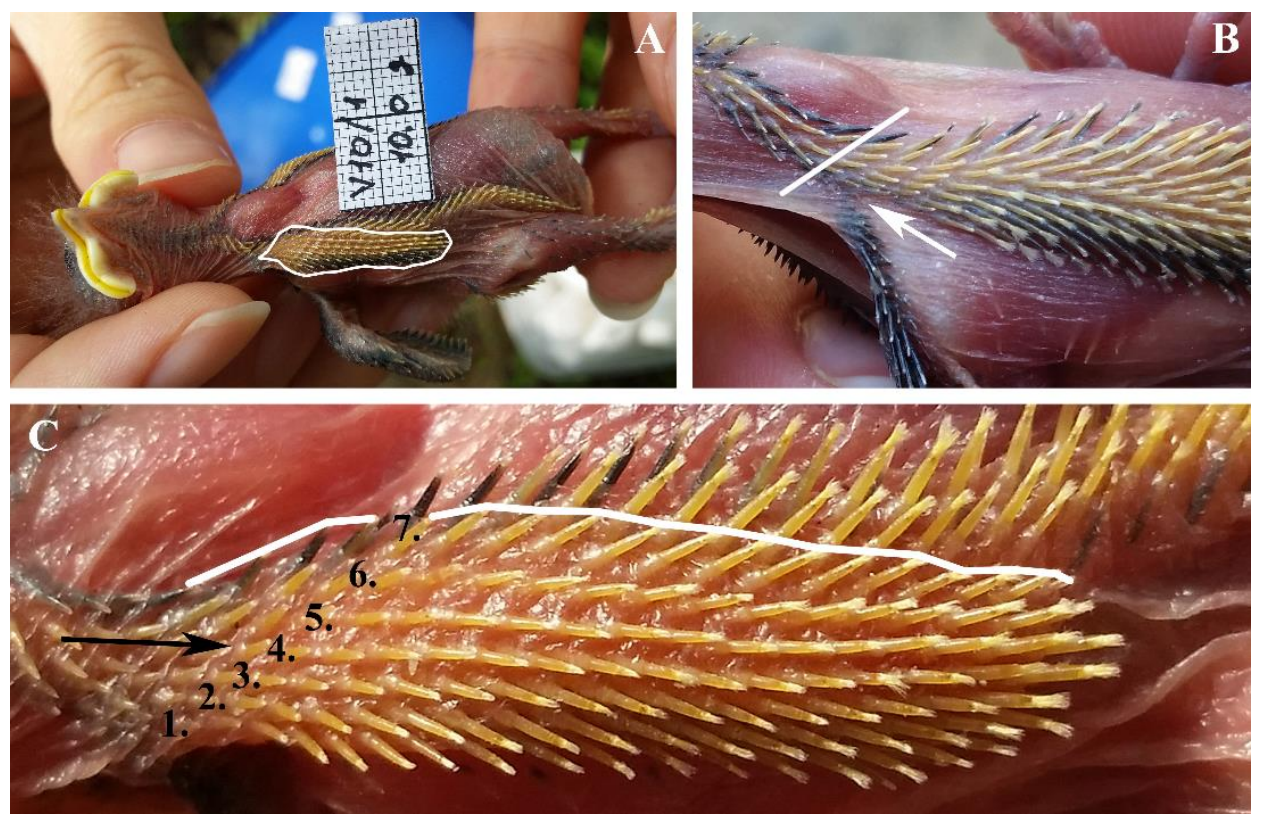
456 Fig. 2. Habitat differences between number (a) and density (b) of contour feathers between 6-9 day old 457 forest and urban Great Tit nestlings.

habitat $\biguplus_{\text {forest }} \xi_{\text {urban }}$

a

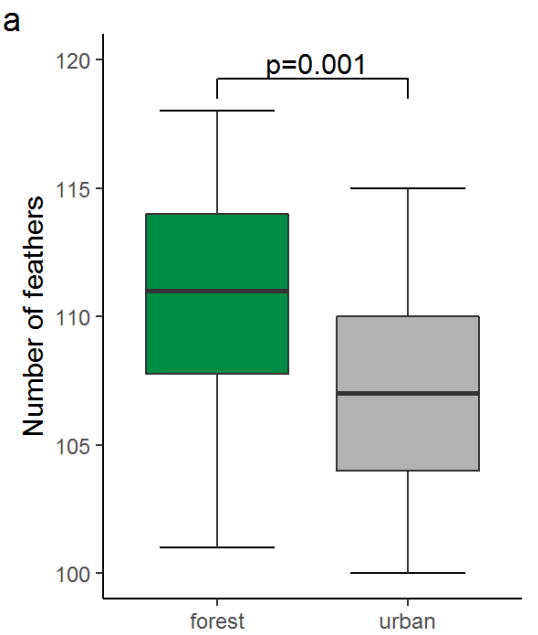

b

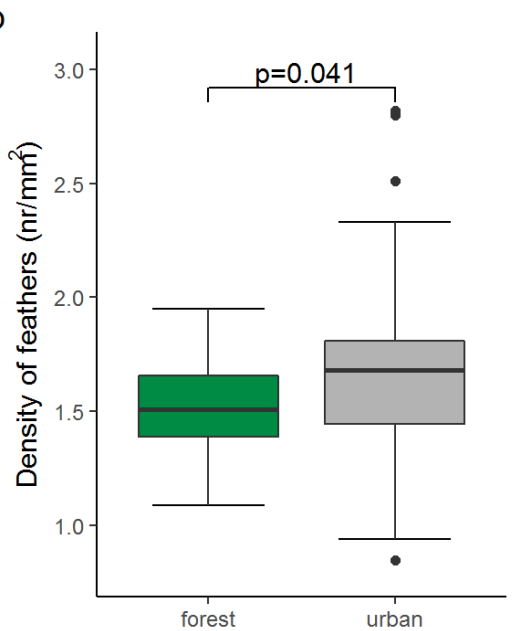

459 